\title{
Characterization of the ATPase Flal of the motor complex of the Pyrococcus furiosus archaellum and its interactions between the ATP-binding protein FlaH
}

\author{
Paushali Chaudhury ${ }^{1}$, Chris van der Does ${ }^{1}$, Sonja-Verena Albers ${ }^{\text {Corresp. } 1}$ \\ ${ }^{1}$ Molecular Biology of Archaea, Institute of Biology, University of Freiburg, Freiburg, Germany \\ Corresponding Author: Sonja-Verena Albers \\ Email address: sonja.albers@biologie.uni-freiburg.de
}

The archaellum, the rotating motility structure of archaea, is best studied in the crenarchaeon Sulfolobus acidocaldarius. To better understand how assembly and rotation of this structure is driven, two ATP-binding proteins, Flal and FlaH of the motor complex of the archaellum of the euryarchaeon Pyrococcus furiosus, were overexpressed, purified and studied. Contrary to the Flal ATPase of $S$. acidocaldarius, which only forms a hexamer after binding of nucleotides, Flal of $P$. furiosusformed a hexamer in a nucleotide independent manner. In this hexamer only 2 of the ATP binding sites were available for binding of the fluorescent ATP-analog MANT-ATP, suggesting a 2-fold symmetry in the hexamer. P.furiosus Flal showed a 250-fold higher ATPase activity than S. acidocaldariusFlal. Interaction studies between the isolated $\mathrm{N}$ - andC-terminal domains of Flal showed interactions between the $\mathrm{N}$ - and $\mathrm{C}$-terminal domains andstrong interactions between the $\mathrm{N}$-terminal domains not previously observed for ATPases involved in archaellum assembly. These interactions played a role in oligomerization and activity, suggesting a conformational state of the hexamer not observed before. Further interaction studies show that the C-terminal domain of PfFlal interacts with the nucleotide binding protein FlaH. This interaction stimulates the ATPase activity of Flal optimally at a 1:1 stoichiometry, suggesting that hexameric PfFlal interacts with hexameric PfFlaH. These data help to further understand the complex interactions that are required to energize the archaellar motor. 
1 Characterization of the ATPase FlaI of the motor complex of the Pyrococcus furiosus archaellum

2 and its interactions between the ATP-binding protein FlaH

3 Paushali Chaudhury ${ }^{1}$, Chris van der Does ${ }^{1}$ and Sonja-Verena Albers ${ }^{1}$

4

$5{ }^{1}$ Molecular Biology of Archaea, Institute of Biology, University of Freiburg, Freiburg, Germany.

6 Corresponding author: Sonja-Verena Albers ${ }^{1}$

7 Email address: sonja.albers@biologie.uni-freiburg.de 


\section{Abstract}

10 The archaellum, the rotating motility structure of archaea, is best studied in the crenarchaeon Sulfolobus acidocaldarius. To better understand how assembly and rotation of this structure is driven, two ATP-binding proteins, FlaI and FlaH of the motor complex of the archaellum of the euryarchaeon Pyrococcus furiosus, were overexpressed, purified and studied. Contrary to the FlaI ATPase of $S$. acidocaldarius, which only forms a hexamer after binding of nucleotides, FlaI of $P$. furiosus formed a hexamer in a nucleotide independent manner. In this hexamer only 2 of the ATP binding sites were available for binding of the fluorescent ATPanalog MANT-ATP, suggesting a 2-fold symmetry in the hexamer. P.furiosus FlaI showed a 250-fold higher ATPase activity than S. acidocaldarius FlaI. Interaction studies between the isolated $\mathrm{N}$ - and $\mathrm{C}$-terminal domains of FlaI showed interactions between the $\mathrm{N}$ - and C-terminal domains and strong interactions between the N-terminal domains not previously observed for ATPases involved in archaellum assembly. These interactions played a role in oligomerization and activity, suggesting a conformational state of the hexamer not observed before. Further interaction studies show that the C-terminal domain of PfFlaI interacts with the nucleotide binding protein FlaH. This interaction stimulates the ATPase activity of FlaI optimally at a 1:1 stoichiometry, suggesting that hexameric PfFlaI interacts with hexameric PfFlaH. These data help to further understand the complex interactions that are required to energize the archaellar motor. 


\section{Introduction}

Motility in archaea is driven by a rotating cell surface appendage, called the archaellum (Jarrell and Albers, 2012; Albers and Jarrell, 2015). The archaellum is widely spread in archaea, and was identified in many of the archaeal phyla, e.g. in crenarchaeota, euryarchaeota, thaumarchaeota and nanoarchaeota (Makarova et al., 2016). Although in function it resembles the bacterial flagellum, it is structurally different as it is evolutionarily related to archaeal and bacterial type IV pilus assembly systems (T4PSs) and Type II secretion systems (T2SS) (Jarrell and Albers, 2012; Berry and Pelicic, 2015; Albers and Jarrell, 2015). The archaellum consists of 7-13 proteins, which are all essential for the assembly and function (Patenge et al., 2001; Thomas et al., 2001; Chaban et al., 2007; Lassak et al., 2012). Similar to the pilins of T4PSs, archaellins also possess N-terminal class III signal peptides, which are processed by a dedicated membrane bound aspartic acid peptidase (Albers et al., 2003; Bardy and Jarrell, 2003). After N-terminal cleavage, the mature archaellins are inserted into the growing archaellum filament. Recently, cryo EM structures of the euryarchaeotes Methanospirillum hungatei and Pyrococcus furiosus archaellum revealed that the archaellin monomer has two domains: an N-terminal domain, which forms a long hydrophobic $\alpha$-helix, and a C-terminal domain with an eight-stranded anti-parallel $\beta$-barrel (Poweleit et al., 2016; Daum et al., 2017). The assembled archaellins showed different inter-subunit interactions than the assembled pilins of T4PSs (Craig et al., 2006; Wang et al., 2017) and the Iho670 adhesion filament of non-motile Ignicoccus hospitalis (Braun et al., 2016). Similar to the assembly of the pilus in T4PS and T2SSs (Jakovljevic et al., 2008; Chiang et al., 2008; Yamagata and Tainer, 2007), assembly of the archaellum is energized by ATP hydrolysis (Thomas et al., 2001; Reindl et al., 2013). Operons encoding components of the archaellum contain two ATP binding proteins, FlaI and FlaH. FlaI of the crenarchaeon Sulfolobus acidocaldarius ( $\mathrm{SaFlaI}$ ) forms an ATP-dependent hexamer and the nucleotide bound crystal structure showed a conserved C-terminal ATPase domain (CTD) which is connected via a flexible linker to the variable N-terminal domain (NTD) (Fig.1A) (Ghosh et al., 2011; Reindl et al., 2013). In the hexameric crystal structure, an intrinsic 2-fold symmetry results in three unique subunit conformations, and superimposition of unique NTDs and CTDs shows that the individual NTD and CTD structures are similar, with only small changes in the NTD. The intra-subunit interface is largest between the CTDs. Indeed, $S a$ FlaI lacking the NTD still exhibits $75 \%$ of the ATPase activity compared to the full-length FlaI (Reindl et al., 2013). In the crystal structures, 
61 the interaction between the NTD and CTD from one subunit is small compared to the interaction 62 between one NTD and the neighboring subunit CTD (Reindl et al., 2013). Together with the hexameric structures of Type II secretion and Type IV pili systems (Yamagata and Tainer, 2007; Kenneth et al., 2007; Misic et al., 2010; Lu et al., 2013; McCallum et al., 2017) a model evolved in which successive rounds of ATP binding, ATP hydrolysis and ADP release in the three unique subunits result in conformational changes of the subunits. Based on the homology of FlaI and FlaJ with the T4PS proteins, it seems likely that FlaI interacts with FlaJ (Chiang et al., 2005; Takhar et al., 2013; Bischof et al., 2016). Indeed, the flexible crown groove (residues 61-128) of the structure of $\mathrm{SaFlaI}$ contains negatively charged amino acid patches which were proposed to interact with the positively charged cytoplasmic loops of $\mathrm{SaFlaJ}$ (Reindl et al., 2013; Banerjee et al., 2013). A similar interaction was recently proposed between the PilB and PilC of Geobacter metallireducens (McCallum et al., 2017). Conformational changes in the membrane platform protein then might result in insertion or extrusion of the pilin or archaellin into the pilus/archaellum (Chang et al., 2016; McCallum et al., 2017). Indeed, the ATPase activity of Myxococcus xanthus PilB is stimulated by interaction with PilC (Bischof et al., 2016). Additional to its assembly, the archaellum also needs to rotate. How the switch between assembly and rotation occurs is currently unknown, but possibly FlaH, a second ATPase only identified in operons encoding the archaellum but not in operons encoding archaeal Type IV pili systems, is involved in this switch (Chaudhury et al., 2016). FlaH belongs to the RecA superfamily of ATPases. S. acidocaldarius FlaH ( $\mathrm{SaFlaH})$ can bind ATP, but is unable to hydrolyze it, most likely due to the presence of a non-canonical walker B motif. The crystal structures of SaFlaH and Methanocaldococcus jannaschii FlaH were solved (Meshcheryakov and Wolf, 2016; Chaudhury et al., 2016). SaFlaI and SaFlaH interact with each other in an ATP dependent manner (Chaudhury et al., 2016). SaFlaI and $\mathrm{SaFlaH}$ were also shown to interact with S. acidocaldarius FlaX (SaFlaX) (Banerjee et al., 2013). FlaX, which was only identified in crenarchaea, contains an N-terminal transmembrane domain and a C-terminal cytoplasmic domain which, for $\mathrm{SaFlaX}$, forms a ring-like oligomeric structure with a diameter of $30 \mathrm{~nm}$ (Banerjee et al., 2012). Deletion of 57 amino acids which correspond to three helices from the Cterminus of $\mathrm{SaFlaX}$ abolished formation of the ring and interaction with FlaI in vitro (Banerjee et al., 2012; Banerjee et al., 2013). Electron microscopy revealed that, in vitro, SaFlaH could assemble as a second ring inside the $S a$ FlaX ring (Chaudhury et al., 2016). Thus, it was proposed 
92 that the central core of the crenarchaeal archaellum is formed by FlaI together with FlaH, FlaX 93 and FlaJ (Banerjee et al., 2013). In addition to these proteins, a minimal functional archaellum requires the FlaF and FlaG proteins. FlaF and FlaG are monotopic membrane proteins where $\mathrm{SaFlaF}$ has a $\beta$-sandwich fold and interacts with S-layer proteins suggesting that it might act as a stator that anchors the rotating archaellum (Banerjee et al., 2015).

Although the archaellum is widely spread among archaea, the archaellum has mainly been biochemically characterized in the crenarchaeote $S$. acidocaldarius. Several differences have been observed between the archaellum systems of crenarchaea and euryarchaea. For example, whereas most species of crenarchaeota contain only one archaellin, euryarchaeota may possess up to five different archaellins (Jarrell and Albers, 2012). In Methanococcus maripaludis, the archaellum showed a hook-like structure which was not observed in a deletion mutant of the minor archaellin flaB3 (Chaban et al., 2007). Furthermore, FlaX was only identified in crenarchaea (Ghosh and Albers, 2011), whereas euryarchaeota contain the flaC, flaD and flaE genes which conversely are not found in crenarchaea (Jarrell and Albers, 2012; Albers and Jarrell, 2015). Finally, many euryarchaea exhibit chemotaxis systems, which have not been identified in crenarchaea till date (Wuichet et al., 2010). In Halobacterium salinarum and Haloferax volcanii, it has been demonstrated that the FlaC, FlaD and FlaE proteins link rotation of the archaellum to the CheY signal transduction cascade and thus to the chemotaxis system (Schlesner et al., 2009; Quax et al., 2018).

Recently, a low resolution image of the archaellar basal body of the euryarchaeote Thermococcus kodakaraensis was obtained by cryo-tomography (Briegel et al., 2017). This structure shows similarities to the structures of bacterial T4P (Chang et al., 2016), but also shows several unique features. For example, a large conical frustum of up to $500 \mathrm{~nm}$ in diameter was observed at the cytosolic base of the structure. The resolution of this structure is however not high enough to distinguish or identify individual components (Briegel et al., 2017). The cryo-EM structure of the P. furiosus archaellum allowed a more detailed view of the archaellum motor complex (Daum et al., 2017). Like in T. kodakaraensis a cone structure is present below the archaellum motor complexes. After modelling the structures of $\mathrm{SaFlaI}$ and $\mathrm{SaFlaH}$ in the densities close to the membrane, several remaining densities were observed. These probably contain the FlaCDE proteins. 
122 In this study, we set out to characterize the motor subunits of the archaellum of Pyrococcus

123 furiosus, an anaerobic, heterotrophic hyperthermophilic euryarchaeote that can grow at

124 temperatures between $70^{\circ} \mathrm{C}$ and $103^{\circ} \mathrm{C}$, and a pH between 5 and 9 (Fiala and Stetter, 1986). P.

125 furiosus contains monopolar polytrichous archaella (Fiala and Stetter, 1986), which it does not

126 only use to swim, but also to form cable-like cell-cell connections to adhere to solid surfaces

127 (Näther et al., 2006). Here, we continue our previous studies on the biochemical characterization 128 of $P$. furiosus FlaI (PfFlaI) and FlaH (PfFlaH).

\section{Materials \& Methods}

130

131

132

133

134

135

136

137

138

139

140

141

142

143

144

145

146

147

148

149

Strains and plasmids. Escherichia coli strains NEB 10-beta (New England BioLabs) and Rosetta (DE3) (Novagen) were used for cloning purposes and overexpression respectively. Genomic DNA of Pyrococcus furiosus DSM 3638 (Robb et al., 2001) was used as a template for PCR reactions. Plasmids and their construction are described in Supplementary Table S1. Primers used are described in Supplementary Table S2. All plasmids sequences were confirmed by PCR and sequencing.

Overproduction and purification. Overproduction and purification of His-tagged $P$. furiosus FlaI (PfFlaI), PfFlaI-NTD, PfFlaI-CTD, and P.furiosus FlaH (PfFlaH) and the PfFlaH (K39A) and PfFlaH (D126N) mutants were performed as described previously (Chaudhury et al., 2016). Overproduction and purification of StrepII-tagged PfFlaI (E336A) were essentially performed the same except that Streptactin column material (IBA GmbH, Göttingen, Germany) was used, and that the protein was eluted with $2.5 \mathrm{mM}$ d-desthiobiotin. Samples were stored at $-80^{\circ} \mathrm{C}$ until use.

Analytical gel filtration. PfFlaI, PfFlaI-NTD and PfFlaI-CTD were concentrated to $1 \mathrm{mg} / \mathrm{ml}$ in buffer containing 20mM Tris $\mathrm{HCl} \mathrm{pH} 8.0,150 \mathrm{mM} \mathrm{NaCl}$ (buffer A) using Amicon concentrators (Millipore) with a $10 \mathrm{kDa}$ cut-off. $500 \mu \mathrm{l}$ of the concentrated samples or $250 \mu 1$ PfFlaI-NTD mixed with $250 \mu \mathrm{l}$ PfFlaI-CTD and applied to Superdex 200 10/300 GL or Superdex 75 10/300 GL size exclusion columns equilibrated with buffer A. Fractions were analyzed on SDS-PAGE. Thyroglobulin (669 kDa), $\gamma$-globulin (158 kDa), ovalbumin (44 kDa), myoglobin (17 kDa) and vitamin $\mathrm{B}_{12}(1.35 \mathrm{kDa})$ were used as size standards. 
150 MANT-ATP binding. Binding of the fluorescent ATP analog 2'-/3'-O-(N-methylanthraniloyl)

151 adenosine 5'-triphosphate (MANT-ATP, JENA biosciences) was detected by titrating PfFlaI (20

$152 \mathrm{nM}, 100 \mathrm{nM}$ and $5 \mu \mathrm{M}$ in buffer A containing $5 \mathrm{mM} \mathrm{MgCl}_{2}$ ) with increasing concentrations of

153 MANT-ATP in a $150 \mu \mathrm{l}$ cuvette at $20^{\circ} \mathrm{C}$ in a Fluoromax-4 fluorimeter (Horiba Scientific).

154 Excitation and emission wavelengths were set to 285 and $450 \mathrm{~nm}$ respectively with slit widths of

$15510 \mathrm{~nm}$. Fluorescence was corrected for MANT-ATP fluorescence in the absence of protein. To

156 determine the binding affinity of ATP, competition assays between MANT-ATP and ATP were

157 performed. Total fluorescence was determined under the conditions described above after

158 addition of increasing amounts of ATP to a solution containing $20 \mathrm{nM}$ PfFlaI and $10 \mathrm{nM}$ of

159 MANT-ATP. The data were fitted with the Hill equation: $\mathrm{F}=\left(\mathrm{F}_{\max }+\left(\mathrm{F}_{\min }{ }^{-}\right.\right.$

$\left.\left.160 \mathrm{~F}_{\max }\right)^{*}[\mathrm{ATP}]^{\mathrm{n}}\right) /\left(\mathrm{IC}_{50}{ }^{\mathrm{n}}+[\mathrm{ATP}]^{\mathrm{n}}\right)$, where $\mathrm{F}=$ Fluorescence, $\mathrm{F}_{\min }=$ minimal fluorescence, $\mathrm{F}_{\max }=$

161 maximal fluorescence, [ATP] is the ATP concentration, $\mathrm{IC}_{50}$ is the ATP concentration where the

162 fluorescence is reduced by half, and $\mathrm{n}=$ Hill coefficient.

163 ATPase Assay. Release of inorganic phosphate after ATP hydrolysis was determined using the

164 Malachite green assay (Lanzetta et al., 1979) by determining the colorimetric change at $620 \mathrm{~nm}$

165 using a Clariostar plate reader (BMG labtech). ATP hydrolysis at different temperatures was

166 determined by incubating $12.5 \mu \mathrm{g} / \mathrm{ml}$ PfFlaI in buffer A containing $5 \mathrm{mM} \mathrm{MgCl}_{2}$ and $1 \mathrm{mM} \mathrm{ATP}$

167 for 5 minutes at different temperatures. ATP hydrolysis at different $\mathrm{pHs}$ was determined by

168 incubating $12.5 \mu \mathrm{g} / \mathrm{ml}$ PfFlaI in different buffers (20 mM citrate ( $\mathrm{pH} 3.0), 20 \mathrm{mM}$ 2-(N-

169 morpholino)ethane sulfonic acid (MES, pH 6.0), 20 mM 4-(2-hydroxyethyl)-1-piperazine ethane

170 sulfonic acid (HEPES, pH 7.2), 20 mM 2-amino-2-hydroxymethyl-propane-1,3-diol (TRIS, pH

1718.0 / 9.5), $20 \mathrm{mM}$ sodium carbonate-bicarbonate ( $\mathrm{pH}$ 10.0)) containing $5 \mathrm{mM} \mathrm{MgCl}_{2}$ and $1 \mathrm{mM}$

172 ATP for 5 minutes at $70^{\circ} \mathrm{C}$. To determine the ATPase activity of PfFlaI at different ATP

173 concentrations, $12.5 \mu \mathrm{g} / \mathrm{ml}$ PfFlaI was incubated for 5 minutes at $70^{\circ} \mathrm{C}$ in buffer A containing

$1745 \mathrm{mM} \mathrm{MgCl}_{2}$ and different ATP concentrations. The curve was fitted to the Michaelis-Menten

175 equation $\left(\mathrm{v}=\mathrm{v}_{\max } *[\mathrm{ATP}] /\left(\mathrm{K}_{\mathrm{m}}+[\mathrm{ATP}]\right)\right)$. The Hill coefficient was determined from the slope

176 of a plot of $\log ([\mathrm{ATP}])$ vs $\log \left(\mathrm{v} /\left(\mathrm{v}_{\max }-\mathrm{v}\right)\right)$. ATPase activity of PfFlaI, PfFlaI-NTD, PfFlaI-CTD

177 and the stoichiometric mixture of PfFlaI-NTD and PfFlaI-CTD was determined by incubating the

178 proteins at a concentration of $12.5 \mu \mathrm{g} / \mathrm{ml}$ in buffer A containing $5 \mathrm{mM} \mathrm{MgCl}_{2}$ at $70^{\circ} \mathrm{C}$. 
179 Microscale thermophoresis. Microscale thermophoresis was performed as described previously 180 (Chaudhury et al., 2016). To determine the binding affinity of CTD and NTD of PfFlaI, $28 \mathrm{nM}$ 181 labeled $P f$ FlaH in buffer A containing $0.05 \%$ (v/v) Tween-20 was titrated with increasing 182 concentrations $(1.3 \mathrm{nM}-22.5 \mu \mathrm{M})$ of $P f$-CTD and $(5.6 \mathrm{nM}-11.6 \mu \mathrm{M}) P f$-NTD of $P f$ FlaI on a 183 Nano Temper Monolith NT.115 Pico instrument. The data were fitted as described previously 184 (Chaudhury et al., 2016).

\section{RESULTS}

186

187

188

189

190

191

192

193

194

195

196

197

198

199

200

201

202

203

204

205

206

207

208

\section{Overproduction, purification and characterization of $\boldsymbol{P}$. furiosus FlaI}

To compare the crenarchaeal and euryarchaeal archaellum, we set out to biochemically characterize the archaellum of Pyrococcus furiosus. The genetic region that encodes the components of the archaellum of $P$. furiosus encodes the major archaellin $(f l a B 0)$, followed by two minor archaellins (flaB1, flaB2) and the flaCDFGHIJ genes (Robb et al., 2001; Näther et al., 2006; Näther-Schindler et al., 2014). Here, we focus on the FlaI and FlaH proteins. Similar to SaFlaI, Pyrococcus furiosus FlaI (PfFlaI) contains both an N-terminal and a C-terminal domain, which show respectively $36 \%$ and $57 \%$ identity with $\mathrm{SaFlaI}$ (See Fig. 1A and 1B). PfFlaI was previously overexpressed and purified (Chaudhury et al., 2016), and is here characterized further. Analysis of the oligomeric state of PfFlaI using size exclusion chromatography showed that PfFlaI eluted at a position corresponding to a hexamer (Fig. 2A and 2B). Thus, in this respect, PfFlaI differs from $\mathrm{SaFlaI}$, which after purification elutes as a monomer and formed a hexamer in the presence of the non-hydrolysable ATP analog adenylyl-imidodiphosphate (AMPPNP) (Ghosh et al., 2011) (Fig. 2A and 2B). The $\mathrm{Abs}_{260} / \mathrm{Abs}_{280}$ ratio of 0.3 suggested that PfFlaI was isolated in the nucleotide free form. Indeed, further purification steps, dialysis or ammonium sulphate precipitation did not result in a change in the $\mathrm{Abs}_{260} / \mathrm{Abs}_{280}$ ratio. To test whether PfFlaI could bind ATP, titrations with the fluorescent ATP analog MANT-ATP were performed. Similar experiments were performed to determine the nucleotide binding affinity of $\mathrm{SaFlaI}$ (Ghosh et al., 2011). For PfFlaI, the fluorescence increased linearly upon addition of MANTATP, until a maximum was reached after which no further increase was observed. This saturation was dependent on the protein concentration, and when $20 \mathrm{nM}, 100 \mathrm{nM}$ or $5 \mu \mathrm{M}$ protein PfFlaI (Fig. 3A) was used, the maximum was reached for all three concentrations at 1/3 of the PfFlaI concentration used, demonstrating that only 2 of the ATP binding sites in the 
209 hexamer are available for binding of MANT-ATP. Indeed, $\mathrm{SaFlaI}$ crystallized as a hexameric

210 ring with an intrinsic 2-fold symmetry resulting in three different conformations of the 211 monomers (Reindl et al., 2013), suggesting that also for PfFlaI such a 2-fold symmetry with

212 three unique subunits occurs. To determine the affinity for ATP, bound MANT-ATP was

213 competed with ATP resulting in an $\mathrm{IC}_{50}$ of $260 \mathrm{nM}$ at $20^{\circ} \mathrm{C}$ (Fig. 3B). The hydrolysis of ATP by

214 PfFlaI was tested at different temperatures and at different pHs (Fig. 3C and 3D) and the highest

215 activity was found at $70^{\circ} \mathrm{C}$ and $\mathrm{pH} 8.0$. Even though $P$. furiosus can live up to $103^{\circ} \mathrm{C}$, the

216 ATPase activity in vitro decreased above $70^{\circ} \mathrm{C}$ suggesting that at temperatures above $70^{\circ} \mathrm{C}$

217 PfFlaI is instable. At $70^{\circ} \mathrm{C}$ and $\mathrm{pH} 8.0$, ATP was hydrolyzed with a $\mathrm{v}_{\max }$ of $8 \mu \mathrm{moles} \mathrm{mg}^{-1} \mathrm{~min}^{-1}$

218 (Chaudhury et al., 2016) and a $\mathrm{K}_{\mathrm{m}}$ of $580 \mu \mathrm{M}$ (Fig. 3E). ATP hydrolysis did not show

219 cooperativity with increasing ATP concentrations (Hill coefficient =0.9) (Fig. 3E, inset). The

220 maximum activity observed for PfFlaI was 250-fold higher than the maximum activity (at pH 6.5

221 and $75^{\circ} \mathrm{C}$ ) observed for $\mathrm{SaFlaI}$ (Chaudhury et al., 2016), and equals a turn-over of $\sim 500$ ATP

$222 \mathrm{~min}^{-1}$.

223

224

225

226

227

228

229

230

231

232

233

234

235

236

237

238

\section{The N-terminal domains of PfFlaI interact with each other and with the C-terminal}

\section{domains to stimulate ATP hydrolysis}

The crystal structures of $S a$ FlaI revealed that, similar to other described T2SS and T4PS ATPases (Robien et al., 2003; Yamagata and Tainer, 2007; Satyshur et al., 2007; Misic et al., 2010; Lu et al., 2013; McCallum et al., 2017), SaFlaI consists of a variable NTD and a CTD that binds and hydrolyses ATP (Reindl et al., 2013). These domains are connected via a short flexible linker (Reindl et al., 2013). The hexameric structure of SaFlaI was only observed in the crystal structure after incubation with AMP-PNP. Since PfFlaI forms a much more stable hexamer, we set out to study the interactions between the NTDs and CTDs of PfFlaI.

Both the NTD (PfFlaI-NTD; $29 \mathrm{kDa}$ ) and the CTD (PfFlaI-CTD; $31 \mathrm{kDa}$ ) of PfFlaI were overexpressed in E. coli, purified, and were then analyzed on analytical size exclusion chromatography (Fig. 4A and 4B). The PfFlaI-CTD eluted as a monomer whereas, contrary to what was expected, the $P f$ FlaI-NTD eluted not only as monomer but also as dimer and possibly a higher order oligomer. This demonstrates that the NTDs of PfFlaI interact with each other and might play a role in the formation of the hexameric ring. Since in the crystal structure of the SaFlaI hexamer the NTDs interact with the CTDs of the neighboring subunit, it was tested 
239 whether also the PfFlaI-NTD and the PfFlaI-CTD interacted. Equimolar concentrations of

240 PfFlaI-NTD and PfFlaI-CTD were mixed, analyzed on analytical size exclusion chromatography.

241 This resulted in a co-elution of the PfFlaI-NTD and PfFlaI-CTD and a shift of especially the

242 elution position of the $P f$ FlaI-CTD, demonstrating an interaction between the $P f$ FlaI-NTD and

243 the PfFlaI-CTD. To test whether interaction between the PfFlaI-NTD and the PfFlaI-CTD

244 influenced the ATPase activity, ATP hydrolysis of the single domains and of the mixed domains

245 was determined (Fig. 4C). In these experiments, ATP hydrolysis was only observed when both

246 the $P f$ FlaI-NTD and $P f$ FlaI-CTD were present, demonstrating that the PfFlaI-NTD can stimulate

247 ATP hydrolysis by the PfFlaI-CTD.

\section{$248 \quad P f$ FlaH stimulates the ATPase activity of PfFlaI}

249 We have overexpressed and purified P. furiosus FlaH (PfFlaH) and used microscale

250 thermophoresis (MST) to show that nucleotide-bound PfFlaH bound to PfFlaI with a $\mathrm{K}_{\mathrm{D}}$ of $1 \mu \mathrm{M}$

251 (Chaudhury et al., 2016). PfFlaH containing mutations in the Walker A (PfFlaH K39A) and

252 Walker B motifs ( $P f$ FlaH D126N) had a strongly reduced affinity for nucleotides, and bound

253 PfFlaI with a strongly reduced affinity, demonstrating that nucleotide binding by $P f F l a H$ is

254 important for its interaction with PfFlaI (Chaudhury et al., 2016). Here, we further investigated

255 the influence of this interaction on the ATPase activity of PfFlaI. As observed, PfFlaI hydrolyzed

256 ATP, whereas for PfFlaH, no ATPase activity could be observed (Fig. 5A, (Chaudhury et al.,

257 2016)). Also no ATP hydrolysis was observed for PfFlaI with an E336A mutation in the Walker

258 B motif, and for the $P f$ FlaHK39A and $P f$ FlaHD126N proteins. Addition of $P f$ FlaH to $P f$ FlaI

259 stimulated the total ATPase activity. To test whether the stimulation of the ATPase is derived

260 from an increase of the activity of PfFlaH or of PfFlaI, different combinations of mutants in

$261 P f$ FlaH and $P f$ FlaI with WT proteins were tested (Fig. 5A). This demonstrated unequivocally that

262 binding of nucleotide bound $P f$ FlaH stimulates the ATPase activity of PfFlaI. To test the

263 stoichiometry of this interaction, $1 \mu \mathrm{M}$ PfFlaI was incubated with increasing concentrations of

$264 P f$ FlaH. A maximal stimulation was observed when $P f$ FlaH was present in stoichiometric

265 amounts to PfFlaI (Fig. 5B). The stimulation decreased when further increasing amounts of

$266 P f$ FlaH were added. Thus $P f$ FlaI and $P f$ FlaH interact in a 1:1 stoichiometry. 
268 Nucleotide-bound $P f$ FlaH can interact with $P f$ FlaI with an affinity of $1 \mu \mathrm{M}$ (Chaudhury et al., 269 2016). To test whether FlaH interacts with the NTD or CTD of FlaI, interaction assays using 270 microscale thermophoresis were performed using PfFlaH and the P. furiosus NTD and CTD of 271 FlaI (Fig. 6). The PfFlaI-CTD and the PfFlaI-NTD interacted with PfFlaH with affinities of 100 $272 \mathrm{nM}$ and $>8 \mu \mathrm{M}$, respectively. Thus, it was concluded that $P f \mathrm{FlaH}$ interacts specifically with the PfFlaI-CTD. This interaction occurs with a 10-fold higher affinity than the interaction between

274

275

276

277

278 full length hexameric PfFlaI and $P f$ FlaH, showing that $P f$ FlaH can interact in different manners with the hexameric PfFlaI and the monomeric PfFlaI-CTD.

\section{Discussion}

Many archaeal cell surface structures are homologous to bacterial T4PSs, which function in cell attachment to surfaces, DNA transport, biofilm formation and motility (Jarrell et al., 2013; Makarova et al., 2016; Chaudhury et al., 2018). Similar to the T2SSs and T4PSs, most of these systems possess a pilin protein, a prepilin peptidase which cleaves at the N-terminus of the prepilin, an ATPase of the superfamily of traffic ATPases, and a membrane platform protein (Peabody, 2003; Nishida and Chen, 2004). The traffic ATPase was suggested to interact with the membrane platform protein for several systems (Reindl et al., 2013; Banerjee et al., 2013; Bischof et al., 2016; Takhar et al., 2013), and it has been proposed that interactions between the processed pilin, the traffic ATPase protein and the platform protein drive the assembly of the pilus (Chang et al., 2016; McCallum et al., 2017). FlaI, the traffic ATPase of the archaellum differs from the other traffic ATPases in the fact that it not only energizes the assembly of the archaella, but also should drive its rotation. Comparison of the different crystal structures of traffic ATPases showed many different conformations of the NTD relative to the CTD, but in general, binding of ATP results in large domain movements bringing the NTD and CTD closer together (Reindl et al., 2013; Lu et al., 2013; Satyshur et al., 2007). Comparison of the conformations in SaFlaI and $V c$ GspE, the traffic ATPase of the T2SS of Vibrio cholerae, showed that in $V c$ GspE, ATP hydrolysis results in an up and down movement of the domains, whereas, $\mathrm{SaFlaI}$ shows a more rotating movement of the domains, possibly explaining the differences between $\mathrm{SaFlaI}$ and other traffic ATPases (Reindl et al., 2013). The rotating movement of the $\mathrm{SaFlaI}$ hexamer is the result of three different alternating conformations which mostly differ in the position of the NTD relative to the CTD. 
298 Here the euryarchaeal PfFlaI was characterized, and compared to the well characterized

299 crenarchaeal $S a$ FlaI. Firstly, it was observed that PfFlaI forms a stable hexamer whereas $S a$ FlaI 300 was a monomer in solution. The $\mathrm{SaFlaI}$ hexamer was observed only after incubation with a non301 hydrolyzable ATP analogue and at high protein concentrations in the crystal structure. In the

302 ADP-bound $\mathrm{SaFlaI}$ crystal structure, 3 different alternating conformations were observed, which 303 all contained a nucleotide. ATP binding assays with PfFlaI showed that only 2 of the 6 positions 304 in the PfFlaI hexamer were accessible for MANT-ATP, suggesting that PfFlaI also contains 305 alternating conformations, and that 4 of the 6 subunits of the hexamer are not accessible to 306 fluorescently labelled nucleotides. ATP hydrolysis by SaFlaI was highly co-operative, whereas 307 ATP hydrolysis by PfFlaI did not show any cooperativity, suggesting that cooperatively occurs 308 during the assembly of the SaFlaI hexamer, while no cooperativity occurs in the assembled 309 PfFlaI hexamer. Indeed, the PfFlaI hexamer is, contrary to SaFlaI, formed in an ATP independent manner. This suggest that PfFlaI and $S a$ FlaI might differ structurally. Another suggestion that PfFlaI and $\mathrm{SaFlaI}$ differ in their structures comes from the experiments with the isolated NTD and CTD. Whereas in the different $\mathrm{SaFlaI}$ crystal structures obtained, interactions are found between the CTDs and between the CTDs and the NTD, no strong interactions were observed between the NTDs. Indeed, $S a$ FlaI lacking the NTD still exhibits $75 \%$ of the ATPase activity compared to the full-length FlaI (Reindl et al., 2013), suggesting that the NTD plays no important role in oligomerization. In PfFlaI however, deletion of the NTD strongly reduces or abolishes oligomerization and ATPase activity. The activity and oligomerization can be partly recovered by the addition of the isolated NTD, suggesting that the NTD for PfFlaI also plays an intrinsic role in oligomerization. Based on the crystal structure of SaFlaI, it could be expected that this is caused by the stabilization of the interaction between 2 CTDs by an NTD, but our observation that the isolated NTDs of PfFlaI also oligomerize suggests that interactions between NTDs might also play a role in the oligomerization and activity of PfFlaI. Strong interactions between NTDs of traffic ATPases, have currently only been observed for the HP0525 traffic ATPase of the Helicobacter pylori type IV secretion system (Yeo et al., 2000).

Next to the characterization $P f$ FlaI, the interaction of $P f$ FlaI with $P f$ FlaH was further analyzed. Binding of nucleotide bound PfFlaH to PfFlaI stimulated its ATPase activity 2-fold, further demonstrating the importance of this interaction. Maximum stimulation was found at a 1:1 stoichometery, suggesting that hexameric PfFlaH interacts with hexameric PfFlaI. Previously, it 
329

330

331

332

333

334

335

336

337

338

339

340

341

342

343

344

345

346

347

348

349

350

351

352

353

354

355

356

357

358

359

was demonstrated that both $\mathrm{SaFlaI}$ and $\mathrm{SaFlaH}$ interact with $\mathrm{SaFlaX}$ (Banerjee et al., 2013). In vitro assembly of the C-terminal domain of $\mathrm{SaFlaX}$ resulted in ring-like structures with 15 - to 23- fold symmetry with widely different diameters (Banerjee et al., 2012). After incubation with $\mathrm{SaFlaH}$, monomeric $\mathrm{SaFlaH}$ particles were observed inside these $\mathrm{SaFlaX}$ rings (Chaudhury et al., 2016). The amount of FlaH bound inside the rings varied with the size of the ring, but in the most occurring rings with a 20- fold symmetry, 9-10 $\mathrm{SaFlaH}$ monomers could be observed (Chaudhury et al., 2016). The size of the in vivo FlaX ring in the S. acidocaldarius archaellum complex is currently still unknown. However, since $S a$ FlaX also interacts with $S a$ FlaI, and a FlaI hexamer interacts with hexameric FlaH, it seems likely that the motor complex in $S$. acidocaldarius consists of hexameric $\mathrm{SaFlaI}$, bound to hexameric $\mathrm{SaFlaH}$, surrounded by a SaFlaX ring. Like all euryarchaea, $P$. furiosus does not encode a FlaX homolog, but encodes the FlaC and FlaD proteins. However, it is currently not known whether they also form a ring-like structure and whether possible interactions with FlaI or FlaH exist. Remarkably, P. furiosus does not encode a chemotaxis system (Maeder et al, 1999) and thus the FlaCDE proteins should not be related to the CheY signal transduction cascade.

It was proposed that the NTD of FlaI interacts with FlaJ (Reindl et al., 2013; Banerjee et al., 2013), making it likely that the CTD domain of FlaI would interact with FlaH, and we found that PfFlaH interacts with the PfFlaI-CTD. This interaction occurs with a 10-fold higher affinity than the interaction between full length $P f F l a I$ and $P f F l a H$. This suggest that the affinity of the interaction between FlaH and FlaI might be modulated by factors that increase or decrease the accessibility of the CTD of FlaI to FlaH, and this might facilitate a switch between assembly and rotation of the archaellum.

\section{Conclusions}

Our results showed that FlaI of $P$. furiosus differs significantly from the extensively studied FlaI of $S$. acidocaldarius. Contrary to FlaI of $S$. acidocaldarius, which only forms a hexamer after binding of nucleotides, FlaI of $P$. furiosus forms a stable hexamer in a nucleotide independent manner. The presence of the stable hexamer allowed us to study nucleotide binding to the hexamer. This showed that only 2 of the 6 ATP binding sites were available for binding of the fluorescent ATP-analog MANT-ATP, suggesting a 2-fold symmetry in the hexamer and further 
360 suggesting that individual proteins in the hexamer alternate between the empty, ATP and ADP

361 bound states. We also identified strong interactions between the N-terminal domains $S$.

362 acidocaldarius FlaI not identified before for S. acidocaldarius FlaI. These interactions played a

363 role in oligomerization and activity, suggesting a conformational state of the hexamer not

364 observed previously. We further showed that interaction between FlaI and FlaH stimulates the

365 ATPase activity of FlaI. This occurs optimally at a 1:1 stoichiometry, suggesting that a FlaI

366 hexamer can interact with six FlaH proteins or with a FlaH hexamer. Further interaction studies

367 showed that FlaH interacts with the C-terminal domain of PfFlaI. 
371

We thank Prof. Carola Hunte for giving access to Nanotemper Monolith NT.115 Pico instrument and Dr. Rashmi Kumariya for providing the data of the $S a$ FlaI gel filtration.

\section{References}

Albers, S.-V., and Jarrell, K.F. (2015) The archaellum: how Archaea swim. Front Microbiol 6: 23.

Albers, S.V., Szabó, Z., and Driessen, A.J.M. (2003) Archaeal homolog of bacterial type IV prepilin signal peptidases with broad substrate specificity. J Bacteriol 185: 3918-3925.

Banerjee, A., Ghosh, A., Mills, D.J., Kahnt, J., Vonck, J., and Albers, S.-V.V. (2012) FlaX, a unique component of the crenarchaeal archaellum, forms oligomeric ring-shaped structures and interacts with the motor ATPase FlaI. J Biol Chem 287: 43322-30.

Banerjee, A., Neiner, T., Tripp, P., and Albers, S.-V. (2013) Insights into subunit interactions in the Sulfolobus acidocaldarius archaellum cytoplasmic complex. FEBS J 280: 6141-9.

Banerjee, A., Tsai, C.L., Chaudhury, P., Tripp, P., Arvai, A.S., Ishida, J.P., Tainer, J.A, and Albers, S.V. (2015) FlaF is a $\beta$-sandwich protein that anchors the archaellum in the archaeal cell envelope by binding the S-layer protein. Structure 23: 863-872.

Bardy, S.L., and Jarrell, K.F. (2003) Cleavage of preflagellins by an aspartic acid signal peptidase is essential for flagellation in the archaeon Methanococcus voltae. Mol Microbiol 50: 1339-1347.

Berry, J.-L.J.L., and Pelicic, V. (2015) Exceptionally widespread nanomachines composed of type IV pilins: the prokaryotic Swiss Army knives. FEMS Microbiol Rev 39: 1-21.

Bischof, L.F., Friedrich, C., Harms, A., Søgaard-Andersen, L., and Does, C. van der (2016) The Type IV Pilus Assembly ATPase PilB of Myxococcus xanthus Interacts with the Inner Membrane Platform Protein PilC and the Nucleotide-binding Protein PilM. J Biol Chem 291: 6946-6957.

Braun, T., Vos, M.R., Kalisman, N., Sherman, N.E., Rachel, R., Wirth, R., Schröder, G.F., and Egelman, E.H. (2016) Archaeal flagellin combines a bacterial type IV pilin domain with an Iglike domain. Proc Natl Acad Sci 113: 10352-10357.

Briegel, A., Oikonomou, C.M., Chang, Y., Kjær, A., Huang, A.N., Kim, K.W., Ghosal, D., Nguyen, H.H., Kenny, D., Orgorzalek Loo, R.R., Gunsalus, R.P., and Jensen, G.J. (2017) Morphology of the archaellar motor and associated cytoplasmic cone in Thermococcus kodakaraensis. EMBO Rep e201744070.

Chaban, B., Ng, S.Y.M., Kanbe, M., Saltzman, I., Nimmo, G., Aizawa, S.I., and Jarrell, K.F. (2007) Systematic deletion analyses of the fla genes in the flagella operon identify several genes essential for proper assembly and function of flagella in the archaeon, Methanococcus maripaludis. Mol Microbiol 66: 596-609. 
407 Chang, Y.W., Rettberg, L.A., Treuner-Lange, A., Iwasa, J., Søgaard-Andersen, L., and Jensen, 408 G.J. (2016) Architecture of the type IVa pilus machine. Science (80- ) 351: aad2001.

409 Chaudhury, P., Neiner, T., D’Imprima, E., Banerjee, A., Reindl, S., Ghosh, A., Arvai, A.S., 410 Mills, D.J., van der Does, C., Tainer, J.A., Vonck, J., and Albers, S.V. (2016) The nucleotide411 dependent interaction of FlaH and FlaI is essential for assembly and function of the archaellum 412 motor. Mol Microbiol, 99: 674-85

413 Chaudhury, P., Quax, T.E.F., and Albers, S.-V. (2018) Versatile cell surface structures of 414 archaea. Mol Microbiol 107: 298-311.

415 Chiang, P., Habash, M., and Burrows, L.L. (2005) Disparate subcellular localization patterns of 416 Pseudomonas aeruginosa Type IV pilus ATPases involved in twitching motility. J Bacteriol 417 187: 829-39.

418 Chiang, P., Sampaleanu, L.M., Ayers, M., Pahuta, M., Howell, P.L., and Burrows, L.L. (2008) 419 Functional role of conserved residues in the characteristics secretion NTPase motifs of the 420 Pseudomonas aeruginosa type IV pilus motor proteins PilB, PilT and PilU. Microbiology 154: $421 \quad 114-126$.

422 Craig, L., Volkmann, N., Arvai, A.S., Pique, M.E., Yeager, M., Egelman, E.H., and Tainer, J.A. 423 (2006) Type IV Pilus Structure by Cryo-Electron Microscopy and Crystallography: Implications 424 for Pilus Assembly and Functions. Mol Cell 23: 651-662.

425 Daum, B., Vonck, J., Bellack, A., Chaudhury, P., Reichelt, R., Albers, S.-V., Rachel, R., and 426 Kühlbrandt, W. (2017) Structure and in situ organisation of the Pyrococcus furiosus archaellum 427 machinery. Elife 6.

428 Fiala, G., and Stetter, K.O. (1986) Pyrococcus furiosus sp. nov. represents a novel genus of 429 marine heterotrophic archaebacteria growing optimally at $100^{\circ} \mathrm{C}$. Arch Microbiol 145: 56-61.

430 Ghosh, A., and Albers, S.-V. (2011) Assembly and function of the archaeal flagellum. Biochem 431 Soc Trans 39: 64-9.

432 Ghosh, A., Hartung, S., Does, C. van der, Tainer, J.A., and Albers, S.-V. (2011) Archaeal 433 flagellar ATPase motor shows ATP-dependent hexameric assembly and activity stimulation by 434 specific lipid binding. Biochem J 437: 43-52.

435 Jakovljevic, V., Leonardy, S., Hoppert, M., and Søgaard-Andersen, L. (2008) PilB and PilT are 436 ATPases acting antagonistically in type IV pilus function in Myxococcus xanthus. J Bacteriol 437 190: 2411-21.

438 Jarrell, K., Ding, Y., Nair, D., and Siu, S. (2013) Surface Appendages of Archaea: Structure, 439 Function, Genetics and Assembly. Life 3: 86-117.

440 Jarrell, K.F., and Albers, S.V. (2012) The archaellum: An old motility structure with a new 441 name. Trends Microbiol 20: 307-312.

442 Satyshur, K.A., Worzalla, G.A., Meyer, L.S., Heiniger, E.K., Aukema, K.G., Misic, A.M., and 443 Forest, K.T. (2007) Crystal Structures of the Pilus Retraction Motor PilT Suggest Large Domain 444 Movements and Subunit Cooperation Drive Motility. Structure 15: 363-376. 
Lanzetta, P.A., Alvarez, L.J., Reinach, P.S., and Candia, O.A. (1979) An improved assay for nanomole amounts of inorganic phosphate. Anal Biochem 100: 95-97.

Lassak, K., Neiner, T., Ghosh, A., Klingl, A., Wirth, R., and Albers, S.V. (2012) Molecular analysis of the crenarchaeal flagellum. Mol Microbiol 83: 110-124.

Lu, C., Turley, S., Marionni, S., Park, Y.-J., Lee, K.K., Patrick, M., Shah, R., Sandkvist, M., Bush, M.F., and Hol, W.G. (2013) Hexamers of the Type II secretion ATPase GspE from Vibrio cholerae with Increased ATPase Activity. Structure 21: 1707-1717.

Maeder, D,L, Weiss, R.B., Dunn, D.M., Cherry, J.L., González, J.M., DiRuggiero, J. and Robb, F.T. (1999) Divergence of the hyperthermophilic archaea Pyrococcus furiosus and $P$. horikoshii inferred from complete genomic sequences. Genetics. 152(4):1299-305.

Makarova, K.S., Koonin, E. V., and Albers, S.-V. (2016) Diversity and Evolution of Type IV pili Systems in Archaea. Front Microbiol 7: 667.

McCallum, M., Tammam, S., Khan, A., Burrows, L.L., and Lynne Howell, P. (2017) The molecular mechanism of the type IVa pilus motors. Nat Commun 8: 15091.

Meshcheryakov, V.A., and Wolf, M. (2016) Crystal structure of the flagellar accessory protein FlaH of Methanocaldococcus jannaschii suggests a regulatory role in archaeal flagellum assembly. Protein Sci 25: 1147-1155.

Misic, A.M., Satyshur, K.A., and Forest, K.T. (2010) P. aeruginosa PilT structures with and without nucleotide reveal a dynamic Type Iv pilus retraction motor. J Biol Chem 400: 10111021.

Näther-Schindler, D.J., Schopf, S., Bellack, A., Rachel, R., and Wirth, R. (2014) Pyrococcus furiosus flagella: Biochemical and transcriptional analyses identify the newly detected flaB0 gene to encode the major flagellin. Front Microbiol 5: 695.

Näther, D.J., Rachel, R., Wanner, G., and Wirth, R. (2006) Flagella of Pyrococcus furiosus: Multifunctional organelles, made for swimming, adhesion to various surfaces, and cell-cell contacts. J Bacteriol 188: 6915-6923.

Nishida, T., and Chen, D.G. (2004) Incorporating spatial autocorrelation into the general linear model with an application to the yellowfin tuna (Thunnus albacares) longline CPUE data. Fish Res 70: 265-274.

Patenge, N., Berendes, A., Engelhardt, H., Schuster, S.C., and Oesterhelt, D. (2001) The fla gene cluster is involved in the biogenesis of flagella in Halobacterium salinarum. Mol Microbiol 41: 653-663.

Peabody, C.R. (2003) Type II protein secretion and its relationship to bacterial type IV pili and archaeal flagella. Microbiology 149: 3051-3072.

Poweleit, N., Ge, P., Nguyen, H.H., Loo, R.R.O., Gunsalus, R.P., and Zhou, Z.H. (2016) CryoEM structure of the Methanospirillum hungatei archaellum reveals structural features distinct from the bacterial flagellum and type IV pilus. Nat Microbiol 2: 16222.

Quax, T.E.F., Altegoer, F., Rossi, F., Li, Z., Rodriguez-Franco, M., Kraus, F., Bange, G., and 
483 Albers, S.V. (2018) Structure and function of the archaeal response regulator CheY. Proc Natl 484 Acad Sci 115: 201716661.

485 Reindl, S., Ghosh, A., Williams, G.J., Lassak, K., Neiner, T., Henche, A.-L., Albers, S.V., and 486 Tainer, J.A. (2013) Insights on FlaI functions in Archaeal Motor Assembly and Motilify from 487 Structures, Conformations and Genetics. Mol Cell 49 (6): 1069-82.

488 Robb, F.T., Maeder, D.L., Brown, J.R., DiRuggiero, J., Stump, M.D., Yeh, R.K., Weiss, R.B., 489 and Dunn, D.M. (2001) Genomic sequence of hyperthermophile, Pyrococcus furiosus:

490 Implications for physiology and enzymology. Methods Enzymol 330: 134-157.

491 Robien, M. a., Krumm, B.E., Sandkvist, M., and Hol, W.G.J. (2003) Crystal Structure of the 492 Extracellular Protein Secretion NTPase EpsE of Vibrio cholerae. J Mol Biol 333: 657-674.

493 Schlesner, M., Miller, A., Streif, S., Staudinger, W.F., Müller, J., Scheffer, B., Siedler, F., and 494 Oesterhelt, D. (2009) Identification of Archaea-specific chemotaxis proteins which interact with 495 the flagellar apparatus. BMC Microbiol 9: 56.

496 Takhar, H.K., Kemp, K., Kim, M., Howell, P.L., and Burrows, L.L. (2013) The platform protein 497 is essential for type IV pilus biogenesis. J Biol Chem 288: 9721-8.

498 Thomas, N.A., Pawson, C.T., and Jarrell, K.F. (2001) Insertional inactivation of the flaH gene in 499 the archaeon Methanococcus voltae results in non-flagellated cells. Mol Genet Genomics 265: $500596-603$.

501 Wang, F., Coureuil, M., Osinski, T., Orlova, A., Altindal, T., Gesbert, G., Nassif, X., Egelman, 502 E.H., and Craig, L. (2017) Cryoelectron Microscopy Reconstructions of the Pseudomonas 503 aeruginosa and Neisseria gonorrhoeae Type IV Pili at Sub-nanometer Resolution. Structure 25: 504 1423-1435.e4.

505 Wuichet, K., Cantwell, B.J., and Zhulin, I.B. (2010) Evolution and phyletic distribution of two506 component signal transduction systems. Curr Opin Microbiol 13: 219-225.

507 Yamagata, A., and Tainer, J.A. (2007) Hexameric structures of the archaeal secretion ATPase

$508 \mathrm{GspE}$ and implications for a universal secretion mechanism. EMBO J 26: 878-890.

509 Yeo, H.J., Savvides, S.N., Herr, A.B., Lanka, E., and Waksman, G. (2000) Crystal structure of 510 the hexameric traffic ATPase of the Helicobacter pylori type IV secretion system. Mol Cell 6:

511 1461-1472.

512

513

514 


\section{Figure 1 (on next page)}

Current models of the euryarchaeal and crenarchaeal archaellum motor complex

In both shown archaellum motor complexes, FlaH, Flal and FlaJ are conserved. FlaB, the archaellin, builds the fiament in the archaeal cell envelope (S-layer). The euryarchaeal archaellum motor complex contains FlaC/D/E which are thought to interact with the chemotaxis system. Additionally, a polar cap structure was identified very recently. However, its function is so far unknown. In the crenarchaeal archaellum motor complex FlaX forms a ring-like structure and is thought to act as a scaffold protein for motor protein assembly. 
S-layer

FlaR Peers

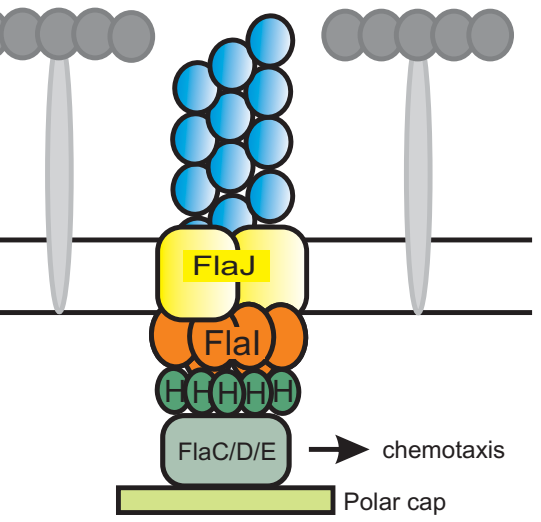

Euryarchaeota

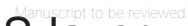

S-layer FlaB

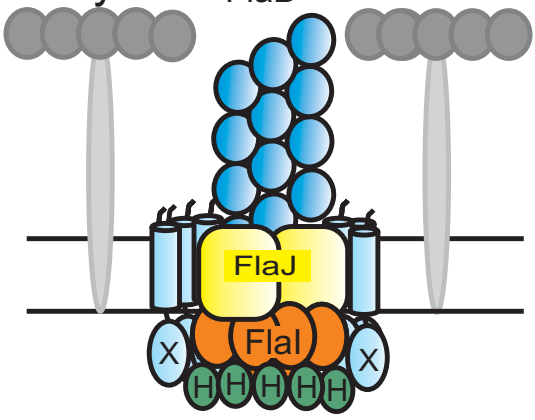

Crenarchaeota 
Figure 2 (on next page)

Pfflal forms a stable hexamer

(A) Relative absorbance at $280 \mathrm{~nm}$ of size exclusion chromatography of PfFlal (64 kDa) shown in black line and SaFlal (59 kDa) is shown in grey line using Superdex 200 10/300GL column. Elution positions of molecular mass standards $[\mathrm{kDa}]$ were indicated with arrows. $(B)$ Coomassie stained SDS-PAGE analysis of the different elution fractions. 

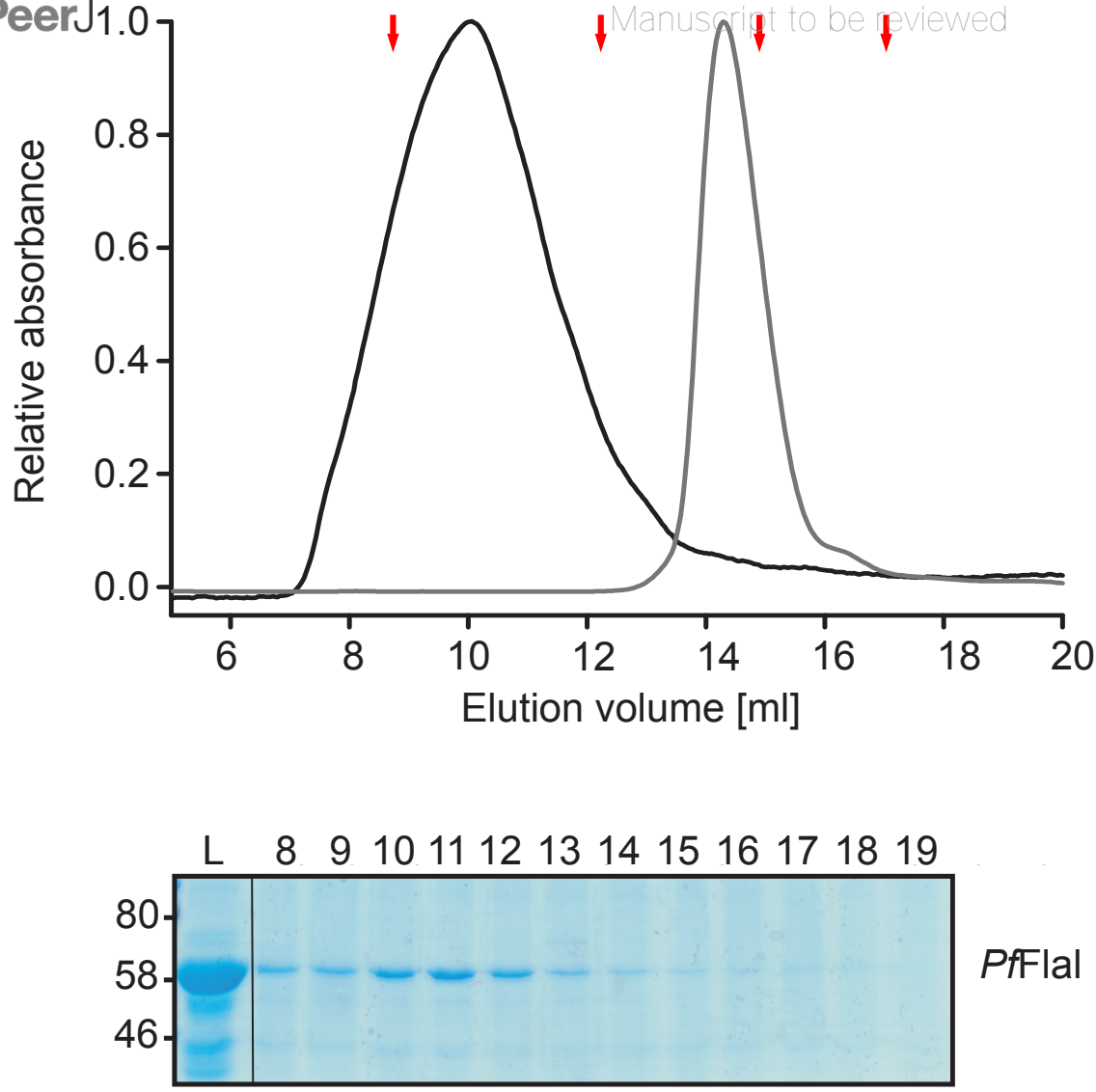

Pfflal

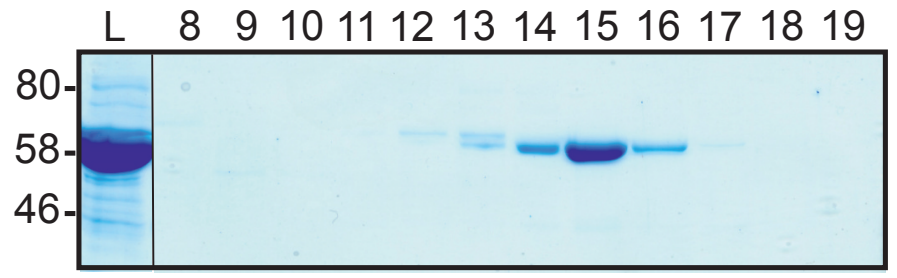

SaFlal 


\section{Figure $\mathbf{3}$ (on next page)}

ATP binding and hydrolysis of PfFlal

(A) Fluorescence increase at increasing concentrations of MANT-ATP upon addition of $20 \mathrm{nM}$, $100 \mathrm{nM}$ and $5 \mu \mathrm{M}$ Pfflal. Lines depict the linear fits of the two observed phases. The lines cross at a MANT-ATP concentration of $1.7 \mu \mathrm{M}$. (B) Total fluorescence after addition of increasing amounts of ATP to a solution containing $20 \mathrm{nM}$ PfFlal and $10 \mathrm{nM}$ of MANT-ATP. The data were fitted with the Hill equation: $\mathrm{F}=\left(\mathrm{F}_{\text {max }}+\left(\mathrm{F}_{\text {min }}-\mathrm{F}_{\text {max }}\right) *[A T P]^{n}\right) /\left(I \mathrm{C}_{50}{ }^{\mathrm{n}}+[\mathrm{ATP}]^{\mathrm{n}}\right)$ resulting in a best fit $\left(R^{2}=0.98\right)$ with $I C_{50}=260 \mathrm{nM}$ and $\mathrm{n}=0.67$. $(C, D)$ ATP hydrolysis by $12.5 \mu \mathrm{g} / \mathrm{ml}$ PfFlal at different temperatures and at different pHs respectively. (E) ATPase activity of PfFlal at different ATP concentrations. The curve was fitted to the Michaelis-Menten equation $(\mathrm{V}=\mathrm{Vmax} *[\mathrm{ATP}] /(\mathrm{Km}+[\mathrm{ATP}]))$, resulting in a $\mathrm{K}_{\mathrm{m}}$ of $580 \mathrm{nM}$. The inset shows the same data plotted according to the Hill equation (Hill coefficient $=0.9$ ). Experiments were performed with at least 2 biological and 3 technical replicates. Error bars depict the standard error obtained from the technical replicates. 


\section{Figure 4 (on next page)}

Analysis of the interaction between the $\mathrm{N}$ - and C-terminal domains of PfFlal

(A) Relative absorbance at $280 \mathrm{~nm}$ of size exclusion chromatography at $280 \mathrm{~nm}$ of size exclusion chromatography of PfFlal-NTD (black line), PfFlal-CTD (grey line) or a stoichiometric mixture of PfFlal-NTD and PfFlal-CTD (dashed line) using Superdex 75 10/300GL column. Elution positions of molecular mass standards [kDa] are indicated with arrows. (B) SDS-PAGE analysis of the elution fractions described in $A$. (C) ATPase activity at $70^{\circ} \mathrm{C}$ of the main elution fraction after size exclusion chromatography of PfFlal, PfFlal-NTD, PfFlal-CTD and the stoichiometric mixture of PfFlal-NTD and PfFlal-CTD at a concentration of $12.5 \mu \mathrm{g} / \mathrm{ml}$. For $A$ and $B$, a representative experiment is shown. For $C$, graph shows the average of 2 biological replicates with 2 technical replicates. 
Figure $\mathbf{5}$ (on next page)

Nucleotide bound PfFlaH stimulates the ATPase activity of PfFlal

(A) The ATPase activity at $70^{\circ} \mathrm{C}$ was determined for PfFlal, PfFlaH and these proteins with mutations in their respective Walker A PfFlaH(K39A) and walker B motifs PfFlal(E336A), PfFlaH(D126N) and of different combinations of these proteins. Proteins were added to a final concentration of $1 \mu \mathrm{M}$. (B) The ATPase activity at $70^{\circ} \mathrm{C}$ was determined for $1 \mu \mathrm{M}$ PfFlal and increasing amounts of PfFlaH, PfFlaH(K39A) and PfFlaH(D126N) shown in solid line, dashed line and dotted line respectively. The graphs show the average of two biological replicates with two technical replicates. 

Figure $\mathbf{6}$ (on next page)

PfFlaH interacts with higher affinity with PfFlal-CTD than PfFlaI-NTD

The binding of $28 \mathrm{nM}$ fluorescently labeled Pf FlaH to increasing concentrations of $1.4 \mathrm{nM}$ $22.5 \mu \mathrm{M}$ for PfFlal-CTD (black) and 5.7nM- $11.7 \mu \mathrm{M}$ for PfFlal-NTD (grey) was studied by microscale thermophoresis. Binding is depicted as the fraction bound and binding curves were fitted to the Hill equation. Curves were obtained from at least two independent experiments. 


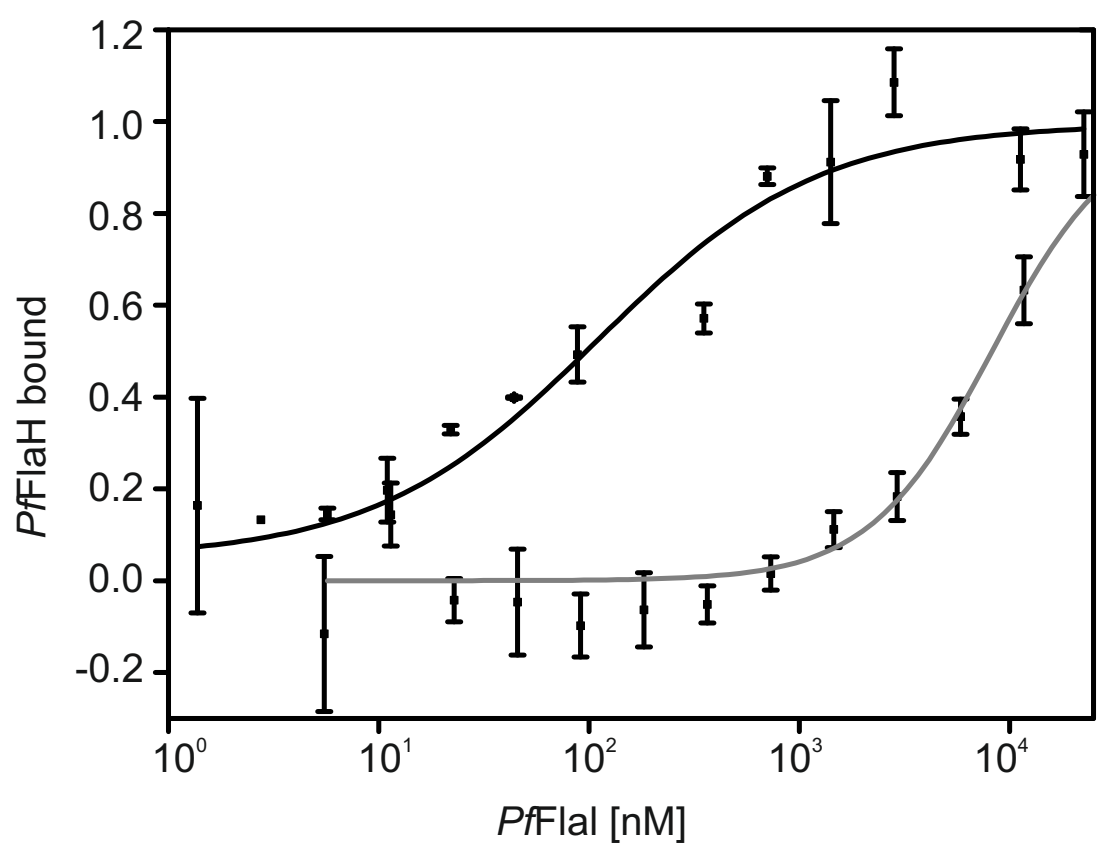

